\title{
Generalized Fractional Integral Operators and Their Commutators with Functions in Generalized Campanato Spaces on Orlicz Spaces
}

\author{
Minglei Shi, Ryutaro Arai and Eiichi Nakai*
}

\begin{abstract}
We investigate the commutators $\left[b, I_{\rho}\right]$ of generalized fractional integral operators $I_{\rho}$ with functions $b$ in generalized Campanato spaces and give a necessary and sufficient condition for the boundedness of the commutators on Orlicz spaces. To do this we define Orlicz spaces with generalized Young functions and prove the boundedness of generalized fractional maximal operators on the Orlicz spaces.
\end{abstract}

\section{Introduction}

Let $\mathbb{R}^{n}$ be the $n$-dimensional Euclidean space, and let $I_{\alpha}$ be the fractional integral operator of order $\alpha \in(0, n)$, that is,

$$
I_{\alpha} f(x)=\int_{\mathbb{R}^{n}} \frac{f(y)}{|x-y|^{n-\alpha}} d y, \quad x \in \mathbb{R}^{n} .
$$

Then it is known as the Hardy-Littlewood-Sobolev theorem that $I_{\alpha}$ is bounded from $L^{p}\left(\mathbb{R}^{n}\right)$ to $L^{q}\left(\mathbb{R}^{n}\right)$, if $\alpha \in(0, n), p, q \in(1, \infty)$ and $-n / p+\alpha=-n / q$. This boundedness was extended to Orlicz spaces by several authors, see [3, 5, 15, 27, 32, 34], etc. Chanillo [2] considered the commutator

$$
\left[b, I_{\alpha}\right] f=b I_{\alpha} f-I_{\alpha}(b f)
$$

with $b \in \mathrm{BMO}$ and proved that $\left[b, I_{\alpha}\right]$ has the same boundedness as $I_{\alpha}$. The result was also extended to Orlicz spaces by Fu, Yang and Yuan [6] and Guliyev, Deringoz and Hasanov [8].

In this paper we consider generalized fractional integral operators $I_{\rho}$ on Orlicz spaces. For a function $\rho:(0, \infty) \rightarrow(0, \infty)$, the operator $I_{\rho}$ is defined by

$$
I_{\rho} f(x)=\int_{\mathbb{R}^{n}} \frac{\rho(|x-y|)}{|x-y|^{n}} f(y) d y, \quad x \in \mathbb{R}^{n},
$$

where we always assume that

$$
\int_{0}^{1} \frac{\rho(t)}{t} d t<\infty .
$$

Received July 23, 2018; Accepted December 18, 2018.

Communicated by Duy-Minh Nhieu.

2010 Mathematics Subject Classification. 46E30, 42B35.

Key words and phrases. Orlicz space, Campanato space, fractional integral, commutator.

${ }^{*}$ Corresponding author. 
If $\rho(r)=r^{\alpha}, 0<\alpha<n$, then $I_{\rho}$ is the usual fractional integral operator $I_{\alpha}$. The condition 1.2 is needed for the integral in 1.1) to converge for bounded functions $f$ with compact support. In this paper we also assume that there exist positive constants $C, K_{1}$ and $K_{2}$ with $K_{1}<K_{2}$ such that, for all $r>0$,

$$
\sup _{r \leq t \leq 2 r} \rho(t) \leq C \int_{K_{1} r}^{K_{2} r} \frac{\rho(t)}{t} d t .
$$

The operator $I_{\rho}$ was introduced in [20] to extend the Hardy-Littlewood-Sobolev theorem to Orlicz spaces whose partial results were announced in [19]. For example, the generalized fractional integral $I_{\rho}$ is bounded from $\exp L^{p}\left(\mathbb{R}^{n}\right)$ to $\exp L^{q}\left(\mathbb{R}^{n}\right)$, where

$$
\rho(r)=\left\{\begin{array}{ll}
1 /(\log (1 / r))^{\alpha+1} & \text { for small } r, \\
(\log r)^{\alpha-1} & \text { for large } r,
\end{array} \quad \alpha>0,\right.
$$

$p, q \in(0, \infty),-1 / p+\alpha=-1 / q$ and $\exp L^{p}\left(\mathbb{R}^{n}\right)$ is the Orlicz space $L^{\Phi}\left(\mathbb{R}^{n}\right)$ with

$$
\Phi(r)= \begin{cases}1 / \exp \left(1 / r^{p}\right) & \text { for small } r \\ \exp \left(r^{p}\right) & \text { for large } r .\end{cases}
$$

See also $21,24,26]$. Recently, in [4] some necessary and sufficient conditions for the boundedness of $I_{\rho}$ on Orlicz spaces have been given.

In this paper we consider the commutator $\left[b, I_{\rho}\right]$ with a function $b$ in generalized Campanato spaces. To prove the boundedness of $\left[b, I_{\rho}\right]$ on Orlicz spaces we need the sharp maximal operator $M^{\sharp}$ and generalized fractional maximal operators $M_{\rho}$, see (1.6) and (1.7) below for their definitions. Moreover, we need a generalization of the Young function.

First we recall the definition of the generalized Campanato space and the sharp maximal and generalized fractional maximal operators. We denote by $B(x, r)$ the open ball centered at $x \in \mathbb{R}^{n}$ and of radius $r$, that is,

$$
B(x, r)=\left\{y \in \mathbb{R}^{n}:|y-x|<r\right\} .
$$

For a measurable set $G \subset \mathbb{R}^{n}$, we denote by $|G|$ and $\chi_{G}$ the Lebesgue measure of $G$ and the characteristic function of $G$, respectively. For a function $f \in L_{\text {loc }}^{1}\left(\mathbb{R}^{n}\right)$ and a ball $B$, let

$$
f_{B}=f_{B} f=f_{B} f(y) d y=\frac{1}{|B|} \int_{B} f(y) d y .
$$

Definition 1.1. For $p \in[1, \infty)$ and $\psi:(0, \infty) \rightarrow(0, \infty)$, let $\mathcal{L}_{p, \psi}\left(\mathbb{R}^{n}\right)$ be the set of all functions $f$ such that the following functional is finite:

$$
\|f\|_{\mathcal{L}_{p, \psi}\left(\mathbb{R}^{n}\right)}=\sup _{B=B(x, r)} \frac{1}{\psi(r)}\left(f_{B}\left|f(y)-f_{B}\right|^{p} d y\right)^{1 / p},
$$

where the supremum is taken over all balls $B(x, r)$ in $\mathbb{R}^{n}$. 
Then $\|f\|_{\mathcal{L}_{p, \psi}\left(\mathbb{R}^{n}\right)}$ is a norm modulo constant functions and thereby $\mathcal{L}_{p, \psi}\left(\mathbb{R}^{n}\right)$ is a Banach space. If $p=1$ and $\psi \equiv 1$, then $\mathcal{L}_{p, \psi}\left(\mathbb{R}^{n}\right)=\operatorname{BMO}\left(\mathbb{R}^{n}\right)$. If $p=1$ and $\psi(r)=r^{\alpha}$ $(0<\alpha \leq 1)$, then $\mathcal{L}_{p, \psi}\left(\mathbb{R}^{n}\right)$ coincides with $\operatorname{Lip}_{\alpha}\left(\mathbb{R}^{n}\right)$.

The sharp maximal operator $M^{\sharp}$ is defined by

$$
M^{\sharp} f(x)=\sup _{B \ni x} f_{B}\left|f(y)-f_{B}\right| d y, \quad x \in \mathbb{R}^{n},
$$

where the supremum is taken over all balls $B$ containing $x$. For a function $\rho:(0, \infty) \rightarrow$ $(0, \infty)$, let

$$
M_{\rho} f(x)=\sup _{B(z, r) \ni x} \rho(r) f_{B(z, r)}|f(y)| d y, \quad x \in \mathbb{R}^{n},
$$

where the supremum is taken over all balls $B(z, r)$ containing $x$. We don't assume the condition (1.2) or 1.3) on the definition of $M_{\rho}$. The operator $M_{\rho}$ was studied in 31] on generalized Morrey spaces. If $\rho(r)=|B(0, r)|^{\alpha / n}$, then $M_{\rho}$ is the usual fractional maximal operator $M_{\alpha}$. If $\rho \equiv 1$, then $M_{\rho}$ is the Hardy-Littlewood maximal operator $M$, that is,

$$
M f(x)=\sup _{B \ni x} f_{B}|f(y)| d y, \quad x \in \mathbb{R}^{n} .
$$

It is known that the usual fractional maximal operator $M_{\alpha}$ is dominated pointwise by the fractional integral operator $I_{\alpha}$, that is, $M_{\alpha} f(x) \leq C I_{\alpha}|f|(x)$ for all $x \in \mathbb{R}^{n}$. Then the boundedness of $M_{\alpha}$ follows from one of $I_{\alpha}$. However, we need a better estimate on $M_{\rho}$ than $I_{\rho}$ to prove the boundedness of the commutator $\left[b, I_{\rho}\right]$. In this paper we give a necessary and sufficient condition of the boundedness of $M_{\rho}$ which sharpens the result in $[4]$.

The organization of this paper is as follows. In Section 2 we recall the definition of the Young function and give its generalization. Then we define Orlicz spaces with generalized Young functions. We state main results in Section 3. We give some lemmas in Section 4 to prove the main results. The boundedness of $I_{\rho}$ has been proved in [4]. We prove the boundedness of $M_{\rho}$ in Section 5. Moreover, we investigate pointwise estimate by using the sharp maximal operator and the norm estimate by the sharp maximal operator in Section 6. Finally, using the generalized Young function and the results in Sections 446 , we prove the boundedness of $\left[b, I_{\rho}\right]$ in Section 7 .

At the end of this section, we make some conventions. Throughout this paper, we always use $C$ to denote a positive constant that is independent of the main parameters involved but whose value may differ from line to line. Constants with subscripts, such as $C_{p}$, are dependent on the subscripts. If $f \leq C g$, we then write $f \lesssim g$ or $g \gtrsim f$; and if $f \lesssim g \lesssim f$, we then write $f \sim g$. 


\section{Generalization of the Young function and Orlicz spaces}

First we define a set $\bar{\Phi}$ of increasing functions $\Phi:[0, \infty] \rightarrow[0, \infty]$ and give some properties of functions in $\bar{\Phi}$.

For an increasing function $\Phi:[0, \infty] \rightarrow[0, \infty]$, let

$$
a(\Phi)=\sup \{t \geq 0: \Phi(t)=0\}, \quad b(\Phi)=\inf \{t \geq 0: \Phi(t)=\infty\}
$$

with convention $\sup \emptyset=0$ and $\inf \emptyset=\infty$. Then $0 \leq a(\Phi) \leq b(\Phi) \leq \infty$. Let $\bar{\Phi}$ be the set of all increasing functions $\Phi:[0, \infty] \rightarrow[0, \infty]$ such that

$$
\begin{gathered}
0 \leq a(\Phi)<\infty, \quad 0<b(\Phi) \leq \infty, \\
\lim _{t \rightarrow+0} \Phi(t)=\Phi(0)=0, \\
\Phi \text { is left continuous on }[0, b(\Phi)), \\
\text { if } b(\Phi)=\infty \text {, then } \lim _{t \rightarrow \infty} \Phi(t)=\Phi(\infty)=\infty, \\
\text { if } b(\Phi)<\infty \text {, then } \lim _{t \rightarrow b(\Phi)-0} \Phi(t)=\Phi(b(\Phi))(\leq \infty) .
\end{gathered}
$$

In what follows, if an increasing and left continuous function $\Phi:[0, \infty) \rightarrow[0, \infty)$ satisfies (2.1) and $\lim _{t \rightarrow \infty} \Phi(t)=\infty$, then we always regard that $\Phi(\infty)=\infty$ and that $\Phi \in \bar{\Phi}$.

For $\Phi \in \bar{\Phi}$, we recall the generalized inverse of $\Phi$ in the sense of O'Neil 27, Definition 1.2].

Definition 2.1. For $\Phi \in \bar{\Phi}$ and $u \in[0, \infty]$, let

$$
\Phi^{-1}(u)= \begin{cases}\inf \{t \geq 0: \Phi(t)>u\} & \text { if } u \in[0, \infty) \\ \infty & \text { if } u=\infty\end{cases}
$$

Let $\Phi \in \bar{\Phi}$. Then $\Phi^{-1}$ is finite, increasing and right continuous on $[0, \infty)$ and positive on $(0, \infty)$. If $\Phi$ is bijective from $[0, \infty]$ to itself, then $\Phi^{-1}$ is the usual inverse function of $\Phi$. Moreover, we have the following proposition, which is a generalization of Property 1.3 in 27 .

Proposition 2.2. Let $\Phi \in \bar{\Phi}$. Then

$$
\Phi\left(\Phi^{-1}(u)\right) \leq u \leq \Phi^{-1}(\Phi(u)) \quad \text { for all } u \in[0, \infty]
$$

Proof. First we show that, for all $t, u \in[0, \infty]$,

$$
\Phi(t) \leq u \quad \Longrightarrow \quad t \leq \Phi^{-1}(u)
$$


If $\Phi(t) \leq u$, then $\Phi(s)>u \Rightarrow \Phi(s)>\Phi(t) \Rightarrow s>t$ and

$$
\{s \geq 0: \Phi(s)>u\} \subset\{s \geq 0: s>t\} .
$$

Hence,

$$
\Phi^{-1}(u)=\inf \{s \geq 0: \Phi(s)>u\} \geq \inf \{s \geq 0: s>t\}=t .
$$

This shows (2.6). Now, letting $\Phi(t)=u$ and using (2.6), we have that $t \leq \Phi^{-1}(u)=$ $\Phi^{-1}(\Phi(t))$, which is the second inequality in 2.5).

Next we show that, for all $t \in(0, \infty]$ and $u \in[0, \infty]$,

$$
\begin{aligned}
\Phi(t)>u & \Longrightarrow t>\Phi^{-1}(u), \\
t \leq \Phi^{-1}(u) & \Longrightarrow \Phi(t) \leq u .
\end{aligned}
$$

We only show (2.7), since 2.8) is equivalent to (2.7). If $\Phi(t)>u$, then $\Phi(s)>u$ for some $s<t$ by the properties (2.2)-(2.4). By the definition of $\Phi^{-1}$ we have that $s \geq \Phi^{-1}(u)$. That is, $t>\Phi^{-1}(u)$, which shows (2.7). Now, if $\Phi^{-1}(u)=0$, then the first inequality in (2.5) is true by 2.1). If $t=\Phi^{-1}(u)>0$, then, using (2.8), we have that $\Phi\left(\Phi^{-1}(u)\right)=$ $\Phi(t) \leq u$, which is the first inequality in 2.5.

For $\Phi, \Psi \in \bar{\Phi}$, we write $\Phi \approx \Psi$ if there exists a positive constant $C$ such that

$$
\Phi\left(C^{-1} t\right) \leq \Psi(t) \leq \Phi(C t) \text { for all } t \in[0, \infty]
$$

For functions $P, Q:[0, \infty] \rightarrow[0, \infty]$, we write $P \sim Q$ if there exists a positive constant $C$ such that

$$
C^{-1} P(t) \leq Q(t) \leq C P(t) \text { for all } t \in[0, \infty]
$$

Then, for $\Phi, \Psi \in \bar{\Phi}$

$$
\Phi \approx \Psi \Longleftrightarrow \Phi^{-1} \sim \Psi^{-1}
$$

Actually we have the following lemma.

Lemma 2.3. Let $\Phi, \Psi \in \bar{\Phi}$, and let $C$ be a positive constant. Then

$$
\Phi(t) \leq \Psi(C t) \quad \text { for all } t \in[0, \infty]
$$

if and only if

$$
\Psi^{-1}(u) \leq C \Phi^{-1}(u) \quad \text { for all } u \in[0, \infty] .
$$

Proof. Let $\Phi(t) \leq \Psi(C t)$ for all $t \in[0, \infty]$. If $t=\Psi^{-1}(u)$, then by Proposition 2.2 we have that $\Psi(t)=\Psi\left(\Psi^{-1}(u)\right) \leq u$ and that

$$
\Psi^{-1}(u) / C=t / C \leq \Phi^{-1}(\Phi(t / C)) \leq \Phi^{-1}(\Psi(t)) \leq \Phi^{-1}(u) .
$$


Conversely, let $\Psi^{-1}(u) \leq C \Phi^{-1}(u)$ for all $u \in[0, \infty]$. If $u=\Psi(t)$, then by Proposition 2.2 we have $t \leq \Psi^{-1}(\Psi(t))=\Psi^{-1}(u)$ and

$$
\Phi(t / C) \leq \Phi\left(\Psi^{-1}(u) / C\right) \leq \Phi\left(\Phi^{-1}(u)\right) \leq u=\Psi(t) .
$$

Next we recall the definition of the Young function and give its generalization.

Definition 2.4. A function $\Phi \in \bar{\Phi}$ is called a Young function (or sometimes also called an Orlicz function) if $\Phi$ is convex on $[0, b(\Phi))$.

By the convexity, any Young function $\Phi$ is continuous on $[0, b(\Phi))$ and strictly increasing on $[a(\Phi), b(\Phi)]$. Hence $\Phi$ is bijective from $[a(\Phi), b(\Phi)]$ to $[0, \Phi(b(\Phi))]$. Moreover, $\Phi$ is absolutely continuous on any closed subinterval in $[0, b(\Phi))$. That is, its derivative $\Phi^{\prime}$ exists a.e. and

$$
\Phi(t)=\int_{0}^{t} \Phi^{\prime}(s) d s, \quad t \in[0, b(\Phi)) .
$$

Definition 2.5. (i) Let $\Phi_{Y}$ be the set of all Young functions.

(ii) Let $\bar{\Phi}_{Y}$ be the set of all $\Phi \in \bar{\Phi}$ such that $\Phi \approx \Psi$ for some $\Psi \in \Phi_{Y}$.

(iii) Let $\mathcal{Y}$ be the set of all Young functions such that $a(\Phi)=0$ and $b(\Phi)=\infty$.

For $\Phi \in \bar{\Phi}_{Y}$, we define the Orlicz space $L^{\Phi}\left(\mathbb{R}^{n}\right)$ and the weak Orlicz space ${ } L^{\Phi}\left(\mathbb{R}^{n}\right)$. Let $L^{0}\left(\mathbb{R}^{n}\right)$ be the set of all complex valued measurable functions on $\mathbb{R}^{n}$.

Definition 2.6. For a function $\Phi \in \bar{\Phi}_{Y}$, let

$$
\begin{aligned}
L^{\Phi}\left(\mathbb{R}^{n}\right) & =\left\{f \in L^{0}\left(\mathbb{R}^{n}\right): \int_{\mathbb{R}^{n}} \Phi(\epsilon|f(x)|) d x<\infty \text { for some } \epsilon>0\right\}, \\
\|f\|_{L^{\Phi}} & =\inf \left\{\lambda>0: \int_{\mathbb{R}^{n}} \Phi\left(\frac{|f(x)|}{\lambda}\right) d x \leq 1\right\} \\
\mathrm{w} L^{\Phi}(\Omega) & =\left\{f \in L^{0}\left(\mathbb{R}^{n}\right): \sup _{t \in(0, \infty)} \Phi(t) m(\epsilon f, t)<\infty \text { for some } \epsilon>0\right\}, \\
\|f\|_{\mathrm{w} L^{\Phi}} & =\inf \left\{\lambda>0: \sup _{t \in(0, \infty)} \Phi(t) m\left(\frac{f}{\lambda}, t\right) \leq 1\right\}
\end{aligned}
$$

where $m(f, t)=\left|\left\{x \in \mathbb{R}^{n}:|f(x)|>t\right\}\right|$.

Then $\|\cdot\|_{L^{\Phi}}$ and $\|\cdot\|_{\mathrm{w} L^{\Phi}}$ are quasi-norms and $L^{\Phi}\left(\mathbb{R}^{n}\right) \subset L_{\text {loc }}^{1}\left(\mathbb{R}^{n}\right)$. If $\Phi \in \Phi_{Y}$, then $\|\cdot\|_{L^{\Phi}}$ is a norm and thereby $L^{\Phi}\left(\mathbb{R}^{n}\right)$ is a Banach space. For $\Phi, \Psi \in \bar{\Phi}_{Y}$, if $\Phi \approx \Psi$, then $L^{\Phi}\left(\mathbb{R}^{n}\right)=L^{\Psi}\left(\mathbb{R}^{n}\right)$ and $\mathrm{w} L^{\Phi}\left(\mathbb{R}^{n}\right)=\mathrm{w} L^{\Psi}\left(\mathbb{R}^{n}\right)$ with equivalent quasi-norms, respectively. Orlicz spaces are introduced by [28, 29]. For the theory of Orlicz spaces, see [14 17,30$]$ for example. 
We note that, for any Young function $\Phi$, we have that

$$
\sup _{t \in(0, \infty)} \Phi(t) m(f, t)=\sup _{t \in(0, \infty)} \operatorname{tm}(\Phi(|f|), t)
$$

and then

$$
\begin{aligned}
\|f\|_{\mathrm{w} L^{\Phi}} & =\inf \left\{\lambda>0: \sup _{t \in(0, \infty)} \Phi(t) m\left(\frac{f}{\lambda}, t\right) \leq 1\right\} \\
& =\inf \left\{\lambda>0: \sup _{t \in(0, \infty)} t m\left(\Phi\left(\frac{|f|}{\lambda}\right), t\right) \leq 1\right\} .
\end{aligned}
$$

For the above equality, see [11, Proposition 4.2] for example.

Definition 2.7. (i) A function $\Phi \in \bar{\Phi}$ is said to satisfy the $\Delta_{2}$-condition, denote $\Phi \in$ $\bar{\Delta}_{2}$, if there exists a constant $C>0$ such that

$$
\Phi(2 t) \leq C \Phi(t) \quad \text { for all } t>0 .
$$

(ii) A function $\Phi \in \bar{\Phi}$ is said to satisfy the $\nabla_{2}$-condition, denote $\Phi \in \bar{\nabla}_{2}$, if there exists a constant $k>1$ such that

$$
\Phi(t) \leq \frac{1}{2 k} \Phi(k t) \text { for all } t>0 .
$$

(iii) Let $\Delta_{2}=\Phi_{Y} \cap \bar{\Delta}_{2}$ and $\nabla_{2}=\Phi_{Y} \cap \bar{\nabla}_{2}$.

Remark 2.8. (i) $\Delta_{2} \subset \mathcal{Y}$ and $\bar{\nabla}_{2} \subset \bar{\Phi}_{Y}$ (see [15, Lemma 1.2.3]).

(ii) Let $\Phi \in \bar{\Phi}_{Y}$. Then $\Phi \in \bar{\Delta}_{2}$ if and only if $\Phi \approx \Psi$ for some $\Psi \in \Delta_{2}$, and, $\Phi \in \bar{\nabla}_{2}$ if and only if $\Phi \approx \Psi$ for some $\Psi \in \nabla_{2}$.

(iii) Let $\Phi \in \Phi_{Y}$. Then $\Phi \in \Delta_{2}$ if and only if $C_{\text {comp }}^{\infty}\left(\mathbb{R}^{n}\right)$ is dense in $L^{\Phi}\left(\mathbb{R}^{n}\right)$, and, $\Phi \in \nabla_{2}$ if and only if the Hardy-Littlewood maximal operator $M$ is bounded on $L^{\Phi}\left(\mathbb{R}^{n}\right)$.

(iv) Let $\Phi \in \Phi_{Y}$. Then $\Phi^{-1}$ satisfies the doubling condition by its concavity, that is,

$$
\Phi^{-1}(u) \leq \Phi^{-1}(2 u) \leq 2 \Phi^{-1}(u) \quad \text { for all } u \in[0, \infty] .
$$

The following theorem is known, see [15, Theorem 1.2.1] for example.

Theorem 2.9. Let $\Phi \in \bar{\Phi}_{Y}$. Then $M$ is bounded from $L^{\Phi}\left(\mathbb{R}^{n}\right)$ to $\mathrm{w} L^{\Phi}\left(\mathbb{R}^{n}\right)$, that is, there exists a positive constant $C_{0}$ such that, for all $f \in L^{\Phi}\left(\mathbb{R}^{n}\right)$,

$$
\|M f\|_{\mathrm{w} L^{\Phi}} \leq C_{0}\|f\|_{L^{\Phi}}
$$

Moreover, if $\Phi \in \bar{\nabla}_{2}$, then $M$ is bounded on $L^{\Phi}\left(\mathbb{R}^{n}\right)$, that is, there exists a positive constant $C_{0}$ such that, for all $f \in L^{\Phi}\left(\mathbb{R}^{n}\right)$,

$$
\|M f\|_{L^{\Phi}} \leq C_{0}\|f\|_{L^{\Phi}} .
$$

See also 3, 12, 13, for the Hardy-Littlewood maximal operator on Orlicz spaces. 


\section{Main results}

The following theorem is an extension of the result in 20] and has been proved in [4] essentially, by using Hedberg's method in [9].

Theorem 3.1. [4] Let $\rho:(0, \infty) \rightarrow(0, \infty)$ satisfy (1.2) and (1.3), and let $\Phi, \Psi \in \bar{\Phi}_{Y}$. Assume that there exists a positive constant $A$ such that, for all $r \in(0, \infty)$,

$$
\int_{0}^{r} \frac{\rho(t)}{t} d t \Phi^{-1}\left(1 / r^{n}\right)+\int_{r}^{\infty} \frac{\rho(t) \Phi^{-1}\left(1 / t^{n}\right)}{t} d t \leq A \Psi^{-1}\left(1 / r^{n}\right) .
$$

Then, for any positive constant $C_{0}$, there exists a positive constant $C_{1}$ such that, for all $f \in L^{\Phi}\left(\mathbb{R}^{n}\right)$ with $f \not \equiv 0$,

$$
\Psi\left(\frac{\left|I_{\rho} f(x)\right|}{C_{1}\|f\|_{L^{\Phi}}}\right) \leq \Phi\left(\frac{M f(x)}{C_{0}\|f\|_{L^{\Phi}}}\right) .
$$

Consequently, $I_{\rho}$ is bounded from $L^{\Phi}\left(\mathbb{R}^{n}\right)$ to $\mathrm{w} L^{\Psi}\left(\mathbb{R}^{n}\right)$. Moreover, if $\Phi \in \bar{\nabla}_{2}$, then $I_{\rho}$ is bounded from $L^{\Phi}\left(\mathbb{R}^{n}\right)$ to $L^{\Psi}\left(\mathbb{R}^{n}\right)$.

Remark 3.2. In [4] the condition that $\Phi, \Psi \in \Phi_{Y}$ was assumed. We can extend it to $\Phi, \Psi \in \bar{\Phi}_{Y}$ as Theorem 3.1. Actually, if 3.1 holds for some $\Phi, \Psi \in \bar{\Phi}_{Y}$, then take $\Phi_{1}, \Psi_{1} \in \Phi_{Y}$ with $\Phi \approx \Phi_{1}$ and $\Psi \approx \Psi_{1}$. Then, instead of $\Phi$ and $\Psi, \Phi_{1}$ and $\Psi_{1}$ satisfy (3.1) for some positive constant $A^{\prime}$ by 2.9 .

Here, we give some examples of the pair of $(\rho, \Phi, \Psi)$ which satisfies the assumption in Theorem 3.1. For other examples, see [21]. See also [18] for the boundedness of $I_{\rho}$ on Orlicz space $L^{\Phi}(\Omega)$ with bounded domain $\Omega \subset \mathbb{R}^{n}$.

Example 3.3. If $\rho(r)=r^{\alpha}, \Phi(t)=t^{p}$ and $\Psi(t)=t^{q}$ with $p, q \in[1, \infty)$ and $0<\alpha<n / p$, then

$$
\int_{0}^{r} \frac{\rho(t)}{t} d t \Phi^{-1}\left(1 / r^{n}\right) \sim \int_{r}^{\infty} \frac{\rho(t) \Phi^{-1}\left(1 / t^{n}\right)}{t} d t \sim r^{\alpha-n / p} \quad \text { and } \quad \Psi^{-1}\left(1 / r^{n}\right)=r^{-n / q} .
$$

In this case,

$$
\text { (3.1) } \Longleftrightarrow r^{\alpha-n / p} \lesssim r^{-n / q}, r \in(0, \infty) \quad \Longleftrightarrow \quad \alpha-n / p=-n / q .
$$

Therefore, the Hardy-Littlewood-Sobolev theorem is a corollary of Theorem 3.1 .

Example 3.4. Let $\rho$ and $\Phi$ be as in (1.4) and in (1.5), respectively, and let $\Psi$ be as in (1.5) with $q$ instead of $p$. Assume that $\alpha, p, q \in(0, \infty)$ and $-1 / p+\alpha=-1 / q$. Then

$$
\int_{0}^{r} \frac{\rho(t)}{t} d t \sim \begin{cases}(\log (1 / r))^{-\alpha} & \text { for small } r>0, \\ (\log r)^{\alpha} & \text { for large } r>0,\end{cases}
$$


and

$$
\Phi^{-1}\left(1 / r^{n}\right) \sim\left\{\begin{array} { l l } 
{ ( \operatorname { l o g } ( 1 / r ) ) ^ { 1 / p } , } \\
{ ( \operatorname { l o g } r ) ^ { - 1 / p } , }
\end{array} \quad \Psi ^ { - 1 } ( 1 / r ^ { n } ) \sim \left\{\begin{array}{ll}
(\log (1 / r))^{1 / q} & \text { for small } r>0 \\
(\log r)^{-1 / q} & \text { for large } r>0
\end{array}\right.\right.
$$

In this case we have

$$
\begin{aligned}
\int_{0}^{r} \frac{\rho(t)}{t} d t \Phi^{-1}\left(1 / r^{n}\right) & \sim \int_{r}^{\infty} \frac{\rho(t) \Phi^{-1}\left(1 / t^{n}\right)}{t} d t \\
& \sim \begin{cases}(\log (1 / r))^{-\alpha+1 / p} & \text { for small } r>0, \\
(\log r)^{\alpha-1 / p} & \text { for large } r>0 .\end{cases}
\end{aligned}
$$

Then the pair $(\rho, \Phi, \Psi)$ satisfies (3.1), that is, $I_{\rho}$ is bounded from $\exp L^{p}\left(\mathbb{R}^{n}\right)$ to $\exp L^{q}\left(\mathbb{R}^{n}\right)$.

Example 3.5. Let $\alpha \in(0, n), p, q \in[1, \infty)$ and $-n / p+\alpha=-n / q$. Let

$$
\rho(r)= \begin{cases}r^{\alpha} & \text { for small } r>0 \\ e^{-r} & \text { for large } r>0\end{cases}
$$

Then

$$
\int_{0}^{r} \frac{\rho(t)}{t} d t \sim \begin{cases}r^{\alpha} & \text { for small } r>0 \\ 1 & \text { for large } r>0\end{cases}
$$

(i) If $\Phi(r)=r^{p}$ and $\Psi(r)=\max \left(r^{p}, r^{q}\right)$, then 3.1 holds. In this case $L^{\Phi}\left(\mathbb{R}^{n}\right)=L^{p}\left(\mathbb{R}^{n}\right)$ and $L^{\Psi}\left(\mathbb{R}^{n}\right)=L^{p}\left(\mathbb{R}^{n}\right) \cap L^{q}\left(\mathbb{R}^{n}\right)$.

(ii) If $\Phi(r)=\max \left(0, r^{p}-1\right)$ and $\Psi(r)=\max \left(0, r^{q}-1\right)$, then (3.1) holds, since

$$
\Phi^{-1}(u) \sim\left\{\begin{array} { l l } 
{ 1 } & { \text { for small } u > 0 , } \\
{ u ^ { 1 / p } } & { \text { for large } u > 0 , }
\end{array} \quad \Phi ^ { - 1 } ( 1 / r ^ { n } ) \sim \left\{\begin{array}{ll}
r^{-n / p} & \text { for small } r>0 \\
1 & \text { for large } r>0 .
\end{array}\right.\right.
$$

In this case $L^{\Phi}\left(\mathbb{R}^{n}\right)=L^{p}\left(\mathbb{R}^{n}\right)+L^{\infty}\left(\mathbb{R}^{n}\right)$ and $L^{\Psi}\left(\mathbb{R}^{n}\right)=L^{q}\left(\mathbb{R}^{n}\right)+L^{\infty}\left(\mathbb{R}^{n}\right)$.

A function $\Phi \in \mathcal{Y}$ is called an $N$-function if

$$
\lim _{t \rightarrow+0} \frac{\Phi(t)}{t}=0, \quad \lim _{t \rightarrow \infty} \frac{\Phi(t)}{t}=\infty .
$$

We say that a function $\theta:(0, \infty) \rightarrow(0, \infty)$ is almost increasing (resp. almost decreasing) if there exists a positive constant $C$ such that, for all $r, s \in(0, \infty)$,

$$
\theta(r) \leq C \theta(s) \quad(\text { resp. } \theta(s) \leq C \theta(r)) \quad \text { if } r<s
$$

Then we have the following corollary. 
Corollary 3.6. Let $1<s<\infty$ and $\rho:(0, \infty) \rightarrow(0, \infty)$. Assume that $\rho$ satisfies (1.2) and that $r \mapsto \rho(r) / r^{n / s-\epsilon}$ is almost decreasing for some positive constant $\epsilon$. Then there exist an $N$-function $\Psi$ and a positive constant $C$ such that, for all $r>0$,

$$
C^{-1} \Psi^{-1}\left(\frac{1}{r^{n}}\right) \leq \frac{1}{r^{n / s}} \int_{0}^{r} \frac{\rho(t)}{t} d t \leq C \Psi^{-1}\left(\frac{1}{r^{n}}\right) .
$$

Moreover, $I_{\rho}$ is bounded from $L^{s}\left(\mathbb{R}^{n}\right)$ to $L^{\Psi}\left(\mathbb{R}^{n}\right)$.

In the above, (3.3) can be shown by the same way as the proof of [1, Theorem 3.5]. The boundedness of $I_{\rho}$ from $L^{s}\left(\mathbb{R}^{n}\right)$ to $L^{\Psi}\left(\mathbb{R}^{n}\right)$ is proven by the following way. First note that $\rho$ satisfies 1.3 by Remark 3.7 below. Let $\Phi(t)=t^{s}$. Then we have

$$
\begin{aligned}
\int_{r}^{\infty} \frac{\rho(t) \Phi^{-1}\left(1 / t^{n}\right)}{t} d t & =\int_{r}^{\infty} \frac{\rho(t) / t^{n / s}}{t} d t \lesssim \frac{\rho(r)}{r^{n / s-\epsilon}} \int_{r}^{\infty} \frac{1}{t^{1+\epsilon}} d t \\
& \sim \frac{\rho(r)}{r^{n / s}} \lesssim \frac{1}{r^{n / s}} \int_{0}^{r} \frac{\rho(t)}{t} d t=\Phi^{-1}\left(\frac{1}{r^{n}}\right) \int_{0}^{r} \frac{\rho(t)}{t} d t
\end{aligned}
$$

where we used (3.4) below for the last inequality. Combining this and (3.3), we have (3.1). Then we have the conclusion by Theorem 3.1 .

Remark 3.7. If $r \mapsto \rho(r) / r^{k}$ is almost decreasing for some positive constant $k$, then $\rho$ satisfies (1.3). Actually,

$$
\sup _{r \leq t \leq 2 r} \rho(t) \sim r^{k} \sup _{r \leq t \leq 2 r} \frac{\rho(t)}{t^{k}} \lesssim r^{k} \int_{r / 2}^{r} \frac{\rho(t)}{t^{k+1}} d t \sim \int_{r / 2}^{r} \frac{\rho(t)}{t} d t .
$$

Next we state the result on the operator $M_{\rho}$ defined by (1.7) in which we don't assume (1.2) or 1.3 .

Theorem 3.8. Let $\rho:(0, \infty) \rightarrow(0, \infty)$, and let $\Phi, \Psi \in \bar{\Phi}_{Y}$.

(i) Assume that there exists a positive constant $A$ such that, for all $r \in(0, \infty)$,

$$
\left(\sup _{0<t \leq r} \rho(t)\right) \Phi^{-1}\left(1 / r^{n}\right) \leq A \Psi^{-1}\left(1 / r^{n}\right) .
$$

Then, for any positive constant $C_{0}$, there exists a positive constant $C_{1}$ such that, for all $f \in L^{\Phi}\left(\mathbb{R}^{n}\right)$ with $f \not \equiv 0$,

$$
\Psi\left(\frac{M_{\rho} f(x)}{C_{1}\|f\|_{L^{\Phi}}}\right) \leq \Phi\left(\frac{M f(x)}{C_{0}\|f\|_{L^{\Phi}}}\right) .
$$

Consequently, $M_{\rho}$ is bounded from $L^{\Phi}\left(\mathbb{R}^{n}\right)$ to $\mathrm{w} L^{\Psi}\left(\mathbb{R}^{n}\right)$. Moreover, if $\Phi \in \bar{\nabla}_{2}$, then $M_{\rho}$ is bounded from $L^{\Phi}\left(\mathbb{R}^{n}\right)$ to $L^{\Psi}\left(\mathbb{R}^{n}\right)$.

(ii) Conversely, if $M_{\rho}$ is bounded from $L^{\Phi}\left(\mathbb{R}^{n}\right)$ to $\mathrm{w}^{\Psi}\left(\mathbb{R}^{n}\right)$, then 3.5 holds for some $A$ and all $r \in(0, \infty)$. 
Remark 3.9. Let $\rho:(0, \infty) \rightarrow(0, \infty)$, and let $\Phi, \Psi \in \bar{\Phi}_{Y}$.

(i) Let $\rho_{1}(r)=\sup _{0<t \leq r} \rho(t)$. Then we conclude from the theorem above that $I_{\rho}$ and $I_{\rho_{1}}$ have the same boundedness, that is, we may assume that $\rho$ is increasing.

(ii) Since $\Phi^{-1}$ is pseudo-concave, $u \mapsto \Phi^{-1}(u) / u$ is almost decreasing, and then $r \mapsto$ $\Phi^{-1}\left(1 / r^{n}\right) r^{n}$ is almost increasing. Therefore, from (3.5) it follows that $r \mapsto \rho(r) / r^{n}$ is dominated by the almost decreasing function $r \mapsto \frac{\Psi^{-1}\left(1 / r^{n}\right)}{\Phi^{-1}\left(1 / r^{n}\right) r^{n}}$.

(iii) In [4], under the conditions that $\Phi, \Psi \in \Phi_{Y}$, that $\rho$ is increasing and that $r \mapsto \rho(r) / r^{n}$ is decreasing, a necessary and sufficient condition for the boundedness of $M_{\rho}$ has been given.

Example 3.10. If $\rho(r)=r^{\alpha}, \Phi(t)=t^{p}$ and $\Psi(t)=t^{q}$ with $p, q \in[1, \infty)$ and $0 \leq \alpha \leq n / p$, then

$$
\rho(r) \Phi^{-1}\left(1 / r^{n}\right) \sim r^{\alpha-n / p} \quad \text { and } \quad \Psi^{-1}\left(1 / r^{n}\right)=r^{-n / q}
$$

In this case,

$$
\text { (3.5) } \Longleftrightarrow r^{\alpha-n / p} \lesssim r^{-n / q}, r \in(0, \infty) \Longleftrightarrow \alpha-n / p=-n / q \text {. }
$$

In this example, if $\alpha=0$, then $M_{\rho}$ is the Hardy-Littlewood maximal operator $M$ and 3.5 $\Leftrightarrow p=q$. If $\alpha-n / p=0$, then $M_{\rho}$ is the fractional maximal operator $M_{\alpha}$ and it is bounded from $L^{p}\left(\mathbb{R}^{n}\right)$ to $L^{\infty}\left(\mathbb{R}^{n}\right)$, since we can take

$$
\Psi(r)=\left\{\begin{array}{ll}
0 & \text { for } r \in[0,1], \\
\infty & \text { for } r \in(1, \infty]
\end{array} \quad \text { and } \quad \Psi^{-1}(r)= \begin{cases}1 & \text { for } r \in[0, \infty) \\
\infty & \text { for } r=\infty\end{cases}\right.
$$

Example 3.11. Let $\Phi$ be as in (1.5), and let $\Psi$ be as in 1.5 with $q$ instead of $p$. Assume that $\alpha \in[0, \infty)$ and $p, q \in(0, \infty)$. Let

$$
\rho(r)= \begin{cases}(\log (1 / r))^{-\alpha} & \text { for small } r>0 \\ (\log r)^{\alpha} & \text { for large } r>0\end{cases}
$$

instead of 1.4). Here, we note that, if $0 \leq \alpha \leq 1$, then $\int_{0}^{1} \frac{\rho(t)}{t} d t=\infty$, that is, $I_{\rho}$ is not well defined, while $M_{\rho}$ is well defined. Actually, $M_{\rho}$ is bounded from $\exp L^{p}\left(\mathbb{R}^{n}\right)$ to $\exp L^{q}\left(\mathbb{R}^{n}\right)$, if $-1 / p+\alpha=-1 / q$ for any $\alpha \in[0, \infty)$, see $(3.2)$ for the inverse functions of $\Phi$ and $\Psi$. Moreover, if $-1 / p+\alpha=0$, then $M_{\rho}$ is bounded from $\exp L^{p}\left(\mathbb{R}^{n}\right)$ to $L^{\infty}\left(\mathbb{R}^{n}\right)$, since we can take $\Psi$ as in (3.7). 
Example 3.12. Assume that $\alpha, q \in[0, \infty)$ and $p \in(1, \infty)$. Let $\rho$ be as in (3.8). Then $M_{\rho}$ is bounded from $L^{p}\left(\mathbb{R}^{n}\right)$ to $L^{p}(\log L)^{p_{1}}\left(\mathbb{R}^{n}\right)$, if $p_{1} / p=\alpha$, where $L^{p}(\log L)^{p_{1}}\left(\mathbb{R}^{n}\right)$ is the Orlicz space $L^{\Phi}\left(\mathbb{R}^{n}\right)$ with

$$
\Phi(r)= \begin{cases}r^{p}(\log (1 / r))^{-p_{1}} & \text { for small } r>0 \\ r^{p}(\log r)^{p_{1}} & \text { for large } r>0 .\end{cases}
$$

In this case we have

$$
\Phi^{-1}\left(1 / r^{n}\right) \sim \begin{cases}r^{-n / p}(\log (1 / r))^{-p_{1} / p} & \text { for small } r>0, \\ r^{-n / p}(\log r)^{p_{1} / p} & \text { for large } r>0 .\end{cases}
$$

In this example, if we take $p=1$, then $M_{\rho}$ is bounded from $L^{1}\left(\mathbb{R}^{n}\right)$ to $\mathrm{w} L^{1}(\log L)^{\alpha}\left(\mathbb{R}^{n}\right)$ which is weak type of $L^{1}(\log L)^{\alpha}\left(\mathbb{R}^{n}\right)$.

Finally, we state the result on the commutator $\left[b, I_{\rho}\right]$. Let

$$
\rho^{*}(r)=\int_{0}^{r} \frac{\rho(t)}{t} d t
$$

Theorem 3.13. Let $\rho, \psi:(0, \infty) \rightarrow(0, \infty)$, and let $\Phi, \Psi \in \bar{\Phi}_{Y}$. Assume that $\rho$ satisfies (1.2). Let $b \in L_{\text {loc }}^{1}\left(\mathbb{R}^{n}\right)$.

(i) Let $\Phi, \Psi \in \bar{\Delta}_{2} \cap \bar{\nabla}_{2}$. Assume that $\psi$ is almost increasing and that $r \mapsto \rho(r) / r^{n-\epsilon}$ is almost decreasing for some $\epsilon \in(0, n)$. Assume also that there exists a positive constant $A$ and $\Theta \in \bar{\nabla}_{2}$ such that, for all $r \in(0, \infty)$,

$$
\begin{gathered}
\int_{0}^{r} \frac{\rho(t)}{t} d t \Phi^{-1}\left(1 / r^{n}\right)+\int_{r}^{\infty} \frac{\rho(t) \Phi^{-1}\left(1 / t^{n}\right)}{t} d t \leq A \Theta^{-1}\left(1 / r^{n}\right), \\
\psi(r) \Theta^{-1}\left(1 / r^{n}\right) \leq A \Psi^{-1}\left(1 / r^{n}\right),
\end{gathered}
$$

and that there exists a positive constant $C_{\rho}$ such that, for all $r, s \in(0, \infty)$,

$$
\left|\frac{\rho(r)}{r^{n}}-\frac{\rho(s)}{s^{n}}\right| \leq C_{\rho}|r-s| \frac{\rho^{*}(r)}{r^{n+1}} \quad \text { if } \frac{1}{2} \leq \frac{r}{s} \leq 2 .
$$

If $b \in \mathcal{L}_{1, \psi}\left(\mathbb{R}^{n}\right)$, then $\left[b, I_{\rho}\right]$ is bounded from $L^{\Phi}\left(\mathbb{R}^{n}\right)$ to $L^{\Psi}\left(\mathbb{R}^{n}\right)$ and there exists a positive constant $C$ such that, for all $f \in L^{\Phi}\left(\mathbb{R}^{n}\right)$,

$$
\left\|\left[b, I_{\rho}\right] f\right\|_{L^{\Psi}} \leq C\|b\|_{\mathcal{L}_{1, \psi}}\|f\|_{L^{\Phi}} .
$$

(ii) Conversely, assume that there exists a positive constant $A$ such that, for all $r \in$ $(0, \infty)$,

$$
\Psi^{-1}\left(1 / r^{n}\right) \leq A r^{\alpha} \psi(r) \Phi^{-1}\left(1 / r^{n}\right)
$$


If $\left[b, I_{\alpha}\right]$ is well defined and bounded from $L^{\Phi}\left(\mathbb{R}^{n}\right)$ to $L^{\Psi}\left(\mathbb{R}^{n}\right)$, then $b$ is in $\mathcal{L}_{1, \psi}\left(\mathbb{R}^{n}\right)$ and there exists a positive constant $C$, independent of $b$, such that

$$
\|b\|_{\mathcal{L}_{1, \psi}} \leq C\left\|\left[b, I_{\alpha}\right]\right\|_{L^{\Phi} \rightarrow L^{\Psi}},
$$

where $\left\|\left[b, I_{\alpha}\right]\right\|_{L^{\Phi} \rightarrow L^{\Psi}}$ is the operator norm of $\left[b, I_{\alpha}\right]$ from $L^{\Phi}\left(\mathbb{R}^{n}\right)$ to $L^{\Psi}\left(\mathbb{R}^{n}\right)$.

Example 3.14. Let $\alpha \in(0, n), \beta \in[0,1]$ and $p, q \in(1, \infty)$, and, let

$$
\rho(r)=r^{\alpha}, \quad \psi(r)=r^{\beta}, \quad \Phi(r)=r^{p}, \quad \Psi(r)=r^{q} .
$$

Assume that $-n / p+\alpha+\beta=-n / q$. Take $\Theta(r)=r^{\widetilde{q}}$ with $-n / \widetilde{q}=-n / p+\alpha$. Then (3.11), (3.12) and (3.13) hold, that is, $\left[b, I_{\alpha}\right]$ is bounded from $L^{p}\left(\mathbb{R}^{n}\right)$ to $L^{q}\left(\mathbb{R}^{n}\right)$, where $b \in \operatorname{Lip}_{\beta}\left(\mathbb{R}^{n}\right)$ if $\beta \in(0,1]$, and $b \in \operatorname{BMO}\left(\mathbb{R}^{n}\right)$ if $\beta=0$ which is Chanillo's result in [2].

Example 3.15. Let $\alpha \in(0, n)$ and $\alpha_{1} \in(-\infty, \infty)$. Let $\beta \in(0,1)$ and $\beta_{1} \in(-\infty, \infty)$, or, let $\beta=0$ and $\beta_{1} \in[0, \infty)$. Let

$$
\rho(r)=\left\{\begin{array}{ll}
r^{\alpha}(\log (1 / r))^{-\alpha_{1}}, \\
r^{\alpha},
\end{array} \quad \psi(r)= \begin{cases}r^{\beta}(\log (1 / r))^{-\beta_{1}} & \text { for } r \in(0,1 / e), \\
r^{\beta}(\log r)^{\alpha_{1}}, & \text { for } r \in[1 / e, e], \\
r^{\beta}(\log r)^{\beta_{1}} & \text { for } r \in(e, \infty) .\end{cases}\right.
$$

Then $\rho^{*} \sim \rho$ and $\rho^{\prime}(t) \sim \rho(t) / t$. In this case $\rho$ satisfies (3.13), since $\rho$ is Lipschitz continuous on $[1 /(2 e), 2 e]$, and, for $r, s \in(0,1 / e] \cup[e, \infty)$, there exists $\theta \in(0,1)$ such that

$$
\left|\frac{\rho(r)}{r^{n}}-\frac{\rho(s)}{s^{n}}\right|=\left.|r-s| \frac{d}{d t}\left(\frac{\rho(t)}{t^{n}}\right)\right|_{t=(1-\theta) r+\theta s}|\lesssim| r-s \mid \frac{\rho(r)}{r^{n+1}} \quad \text { if } \frac{1}{2} \leq \frac{r}{s} \leq 2 .
$$

Let $p, q \in(1, \infty)$ and $p_{1}, q_{1} \in(-\infty, \infty)$, and let

$$
\Phi(r)=\left\{\begin{array}{ll}
r^{p}(\log (1 / r))^{-p_{1}}, \\
r^{p}(\log r)^{p_{1}},
\end{array} \Psi(r)= \begin{cases}r^{q}(\log (1 / r))^{-q_{1}} & \text { for small } r>0, \\
r^{q}(\log r)^{q_{1}} & \text { for large } r>0 .\end{cases}\right.
$$

For the inverse functions of $\Phi$ and $\Psi$, see $(3.9)$. If

$$
-n / p+\alpha+\beta=-n / \widetilde{p}+\beta=-n / q, \quad p_{1} / p+\alpha_{1}+\beta_{1}=\widetilde{p}_{1} / \widetilde{p}+\beta_{1}=q_{1} / q
$$

and

$$
\Theta(r)= \begin{cases}r^{\widetilde{p}}(\log (1 / r))^{-\widetilde{p}_{1}} & \text { for small } r>0 \\ r^{\widetilde{p}}(\log r)^{\widetilde{p}_{1}} & \text { for large } r>0\end{cases}
$$

then

$$
\int_{0}^{r} \frac{\rho(t)}{t} d t \Phi^{-1}\left(1 / r^{n}\right) \sim \int_{r}^{\infty} \frac{\rho(t) \Phi^{-1}\left(1 / t^{n}\right)}{t} d t \sim \Theta^{-1}\left(r^{-n}\right)
$$

and

$$
\psi(r) \Theta^{-1}\left(r^{-n}\right) \sim \Psi^{-1}\left(r^{-n}\right) \sim \begin{cases}r^{-n / p+\alpha+\beta}(\log (1 / r))^{-\left(p_{1} / p+\alpha_{1}+\beta_{1}\right)} & \text { for small } r>0, \\ r^{-n / p+\alpha+\beta}(\log r)^{p_{1} / p+\alpha_{1}+\beta_{1}} & \text { for large } r>0 .\end{cases}
$$

In this case $\left[b, I_{\rho}\right]$ is bounded from $L^{p}(\log L)^{p_{1}}\left(\mathbb{R}^{n}\right)$ to $L^{q}(\log L)^{q_{1}}\left(\mathbb{R}^{n}\right)$. 


\section{Lemmas}

In this section we prepare some lemmas to prove our main results.

For a Young function $\Phi$, its complementary function is defined by

$$
\widetilde{\Phi}(t)= \begin{cases}\sup \{t u-\Phi(u): u \in[0, \infty)\} & \text { if } t \in[0, \infty) \\ \infty & \text { if } t=\infty\end{cases}
$$

Then $\widetilde{\Phi}$ is also a Young function and Young's inequality

$$
t u \leq \Phi(t)+\widetilde{\Phi}(u), \quad t, u \in[0, \infty)
$$

holds. It is also known that

$$
t \leq \Phi^{-1}(t) \widetilde{\Phi}^{-1}(t) \leq 2 t, \quad t \geq 0 .
$$

From Young's inequality we have a generalized Hölder's inequality:

$$
\int_{\mathbb{R}^{n}}|f(x) g(x)| d x \leq 2\|f\|_{L^{\Phi}}\|g\|_{L^{\widetilde{\Phi}}}
$$

(see [35, Theorem 6] and [27, Theorem 2.3]).

Lemma 4.1. Let $\Phi \in \Phi_{Y}$. For a measurable set $G \subset \mathbb{R}^{n}$ with finite measure,

$$
\left\|\chi_{G}\right\|_{L^{\Phi}}=\left\|\chi_{G}\right\|_{\mathrm{w} L^{\Phi}}=\frac{1}{\Phi^{-1}(1 /|G|)} .
$$

From 4.1 it follows that, for the characteristic function $\chi_{B}$ of the ball $B$,

$$
\left\|\chi_{B}\right\|_{L^{\widetilde{\Phi}}}=\frac{1}{\widetilde{\Phi}^{-1}(1 /|B|)} \leq|B| \Phi^{-1}(1 /|B|) .
$$

Lemma 4.2. [1] Let $k>0$ and $\rho:(0, \infty) \rightarrow(0, \infty)$. Assume that $\rho$ satisfies (1.2). Let $\rho^{*}$ be as in (3.10). If $r \mapsto \rho(r) / r^{k}$ is almost decreasing, then $r \mapsto \rho^{*}(r) / r^{k}$ is also almost decreasing.

Remark 4.3. Since $\rho^{*}$ is increasing with respect to $r$, if $r \mapsto \rho(r) / r^{k}$ is almost decreasing for some $k>0$, then we see that $\rho^{*}$ satisfies the doubling condition, that is, there exists a positive constant $C$ such that, for all $r \in(0, \infty)$,

$$
\rho^{*}(r) \leq \rho^{*}(2 r) \leq C \rho^{*}(r)
$$

Lemma 4.4. If $\Phi \in \Delta_{2}$, then its derivative $\Phi^{\prime}$ satisfies

$$
\Phi^{\prime}(2 t) \leq C_{\Phi} \Phi^{\prime}(t) \quad \text { a.e. } t \in[0, \infty),
$$

where the constant $C_{\Phi}$ is independent of $t$. 
Proof. From the convexity of $\Phi$ and $\Phi(0)=0$ it follows that its right derivative $\Phi_{+}^{\prime}(t)$ exists for all $t \in[0, \infty)$ and it is increasing. By $(2.10)$ we have

$$
\Phi(t)=\int_{0}^{t} \Phi^{\prime}(s) d s=\int_{0}^{t} \Phi_{+}^{\prime}(s) d s
$$

since $\Phi^{\prime}=\Phi_{+}^{\prime}$ a.e. Then, for all $t \in(0, \infty)$,

$$
\Phi_{+}^{\prime}(2 t) \leq \frac{1}{t} \int_{2 t}^{3 t} \Phi_{+}^{\prime}(s) d s \leq \frac{1}{t} \Phi(3 t) \leq \frac{C_{\Phi}}{t} \Phi(t) \leq C_{\Phi} \Phi_{+}^{\prime}(t) .
$$

This shows the conclusion.

Lemma 4.5. If $\Phi \in \bar{\nabla}_{2}$, then $\Phi\left((\cdot)^{\theta}\right) \in \bar{\nabla}_{2}$ for some $\theta \in(0,1)$.

Proof. If $\Phi \in \bar{\nabla}_{2}$, then there exists a constant $k>1$ such that

$$
\Phi(t) \leq \frac{1}{2 k} \Phi(k t) .
$$

Take $\theta \in(0,1)$ such that $k^{2(1 / \theta-1)} \leq 2$. Then $k^{2} \leq\left(2 k^{2}\right)^{\theta}$ and

$$
\Phi\left(t^{\theta}\right) \leq \frac{1}{2 k} \Phi\left(k t^{\theta}\right) \leq \frac{1}{(2 k)^{2}} \Phi\left(k^{2} t^{\theta}\right) \leq \frac{1}{2\left(2 k^{2}\right)} \Phi\left(\left(2 k^{2} t\right)^{\theta}\right) .
$$

That is, $\Phi\left((\cdot)^{\theta}\right) \in \bar{\nabla}_{2}$.

Remark 4.6. There exists $\Phi \in \nabla_{2}$ such that $\Phi\left((\cdot)^{\theta}\right) \notin \Phi_{Y}$ for any $\theta \in(0,1)$. Actually, let

$$
\Phi(r)=\max \left(r^{2}, 3 r-2\right)= \begin{cases}r^{2} & \text { if } 0 \leq r \leq 1 \\ 3 r-2 & \text { if } 1<r<2, \\ r^{2} & \text { if } 2 \leq r .\end{cases}
$$

Then $\Phi$ is convex and satisfies 2.11) with $k=8$. However, $3 r^{\theta}-2$ is not convex for any $\theta \in(0,1)$.

\section{Proof of Theorem 3.8}

In this section we prove Theorem 3.8 .

Proof of Theorem 3.8(i). We may assume that $\Phi, \Psi \in \Phi_{Y}$ by 2.9$)$. Let $f \in L^{\Phi}\left(\mathbb{R}^{n}\right)$. We may also assume that $\|f\|_{L^{\Phi}}=1$ and $M f(x)>0$ for all $x \in \mathbb{R}^{n}$. For any $x \in \mathbb{R}^{n}$ and any ball $B=B(z, r) \ni x$, if

$$
\Phi\left(\frac{M f(x)}{C_{0}}\right) \geq \frac{1}{r^{n}}
$$


then, by (4.2), $\|f\|_{L^{\Phi}}=1$, 4.3), the doubling condition of $\Phi^{-1}$ and (3.5), we have

$$
\begin{aligned}
\rho(r) f_{B}|f| & \leq 2 \frac{\rho(r)}{|B|}\left\|\chi_{B}\right\|_{L^{\widetilde{\Phi}}} \leq 2 \frac{\rho(r)}{|B|}|B| \Phi^{-1}\left(\frac{1}{|B|}\right) \\
& \lesssim \rho(r) \Phi^{-1}\left(\frac{1}{r^{n}}\right) \leq A \Psi^{-1}\left(\frac{1}{r^{n}}\right) \leq A \Psi^{-1}\left(\Phi\left(\frac{M f(x)}{C_{0}}\right)\right) .
\end{aligned}
$$

Conversely, if

$$
\Phi\left(\frac{M f(x)}{C_{0}}\right) \leq \frac{1}{r^{n}}
$$

then, choosing $t_{0} \geq r$ such that

$$
\Phi\left(\frac{M f(x)}{C_{0}}\right)=\frac{1}{t_{0}^{n}}
$$

and using (3.5) and $(2.5)$, we have

$$
\rho(r) \leq \sup _{0<t \leq t_{0}} \rho(t) \leq A \frac{\Psi^{-1}\left(\Phi\left(\frac{M f(x)}{C_{0}}\right)\right)}{\Phi^{-1}\left(\Phi\left(\frac{M f(x)}{C_{0}}\right)\right)} \leq A \frac{\Psi^{-1}\left(\Phi\left(\frac{M f(x)}{C_{0}}\right)\right)}{\frac{M f(x)}{C_{0}}},
$$

which implies

$$
\rho(r) f_{B}|f| \leq A C_{0} \frac{\Psi^{-1}\left(\Phi\left(\frac{M f(x)}{C_{0}}\right)\right)}{M f(x)} f_{B}|f| \leq A C_{0} \Psi^{-1}\left(\Phi\left(\frac{M f(x)}{C_{0}}\right)\right) .
$$

Hence, we have

$$
M_{\rho} f(x) \leq C_{1} \Psi^{-1}\left(\Phi\left(\frac{M f(x)}{C_{0}}\right)\right),
$$

which shows 3.6 by 2.5 .

To prove Theorem 3.8(ii) we need the following lemma.

Lemma 5.1. Let $\rho:(0, \infty) \rightarrow(0, \infty)$. Then, for all $x \in \mathbb{R}^{n}$ and $r \in(0, \infty)$,

$$
\left(\sup _{0<t \leq r} \rho(t)\right) \chi_{B(0, r)}(x) \leq\left(M_{\rho} \chi_{B(0, r)}\right)(x) .
$$

Proof. Let $x \in B(0, r)$. If $t \leq r$, then we can choose a ball $B(z, t)$ such that $x \in B(z, t) \subset$ $B(0, r)$. Hence,

$$
\rho(t)=\rho(t) f_{B(z, t)} \chi_{B(0, r)}(y) d y \leq\left(M_{\rho} \chi_{B(0, r)}\right)(x) .
$$

Therefore, we have 5.1 .

Proof of Theorem 3.8(ii). By Lemma 5.1 and the boundedness of $M_{\rho}$ from $L^{\Phi}\left(\mathbb{R}^{n}\right)$ to $\mathrm{w} L^{\Psi}\left(\mathbb{R}^{n}\right)$ we have

$$
\left(\sup _{0<t \leq r} \rho(t)\right)\left\|\chi_{B(0, r)}\right\|_{\mathrm{w} L^{\Psi}} \leq\left\|M_{\rho} \chi_{B(0, r)}\right\|_{\mathrm{w} L^{\Psi}} \lesssim\left\|\chi_{B(0, r)}\right\|_{L^{\Phi}} .
$$

Then, by Lemma 4.1 and the doubling condition of $\Phi^{-1}$ and $\Psi^{-1}$ we have the conclusion. 


\section{Sharp maximal operators}

In this section, to prove Theorem 3.13, we prove two propositions involving the sharp maximal operator $M^{\sharp}$ defined by 1.6 .

First we state the John-Nirenberg type theorem for the Campanato space, which is known by 25, Theorem 3.1] for spaces of homogeneous type. See also 11 for its proof in the case of $\mathbb{R}^{n}$.

Theorem 6.1. Let $p \in(1, \infty)$ and $\psi:(0, \infty) \rightarrow(0, \infty)$. Assume that $\psi$ is almost increasing. Then $\mathcal{L}_{p, \psi}\left(\mathbb{R}^{n}\right)=\mathcal{L}_{1, \psi}\left(\mathbb{R}^{n}\right)$ with equivalent norms.

Proposition 6.2. Assume that $\rho:(0, \infty) \rightarrow(0, \infty)$ satisfies $(1.2)$. Let $\rho^{*}(r)$ be as in (3.10). Assume that $\psi$ is almost increasing, that $r \mapsto \rho(r) / r^{n-\epsilon}$ is almost decreasing for some $\epsilon>0$ and that the condition (3.13) holds. Then, for any $\eta \in(1, \infty)$, there exists a positive constant $C$ such that, for all $b \in \mathcal{L}_{1, \psi}\left(\mathbb{R}^{n}\right), f \in C_{\text {comp }}^{\infty}\left(\mathbb{R}^{n}\right)$ and $x \in \mathbb{R}^{n}$,

$$
M^{\sharp}\left(\left[b, I_{\rho}\right] f\right)(x) \leq C\|b\|_{\mathcal{L}_{1, \psi}}\left(\left(M_{\psi^{\eta}}\left(\left|I_{\rho} f\right|^{\eta}\right)(x)\right)^{1 / \eta}+\left(M_{\left(\rho^{*} \psi\right)^{\eta}}\left(|f|^{\eta}\right)(x)\right)^{1 / \eta}\right) .
$$

To prove the proposition we need the following known lemma, for its proof, see Lemma 4.7 and Remark 4.1 in [1] for example.

Lemma 6.3. Let $p \in[1, \infty)$. Assume that $\psi$ is almost increasing. Then there exists a positive constant $C$ such that, for all $f \in \mathcal{L}_{1, \psi}, x \in \mathbb{R}^{n}$ and $r, s \in(0, \infty)$,

$$
\left(f_{B(x, s)}\left|f(y)-f_{B(x, r)}\right|^{p} d y\right)^{1 / p} \leq C\left(1+\log _{2} \frac{s}{r}\right) \psi(s)\|f\|_{\mathcal{L}_{1, \psi}} \quad \text { if } r \leq s .
$$

Proof of Proposition 6.2. For any ball $B=B(x, t)$, let $f=f_{1}+f_{2}$ with $f_{1}=f \chi_{2 B}$, and let

$F_{1}(y)=\left(b(y)-b_{2 B}\right) I_{\rho} f(y), \quad F_{2}(y)=I_{\rho}\left(\left(b-b_{2 B}\right) f_{1}\right)(y), \quad F_{3}(y)=I_{\rho}\left(\left(b-b_{2 B}\right) f_{2}\right)(y)-C_{B}$

for $y \in B$, where $C_{B}=I_{\rho}\left(\left(b-b_{2 B}\right) f_{2}\right)(x)$ and

$$
I_{\rho}\left(\left(b-b_{2 B}\right) f_{2}\right)(y)=\int_{\mathbb{R}^{n}} \frac{\rho(|y-z|)}{|y-z|^{n}}\left(b(z)-b_{2 B}\right) f_{2}(z) d z, \quad y \in B .
$$

Then we have

$$
\left[b, I_{\rho}\right] f+C_{B}=\left[b-b_{2 B}, I_{\rho}\right] f+C_{B}=F_{1}-F_{2}-F_{3} .
$$

We show that

$$
f_{B}\left|F_{i}(y)\right| d y \leq C\|b\|_{\mathcal{L}_{1, \psi}}\left(\left(M_{\psi^{\eta}}\left(\left|I_{\rho} f\right|^{\eta}\right)(x)\right)^{1 / \eta}+\left(M_{\left(\rho^{*} \psi\right)^{\eta}}\left(|f|^{\eta}\right)(x)\right)^{1 / \eta}\right), \quad i=1,2,3 .
$$


Then we have the conclusion.

Now, by Hölder's inequality with $1 / \eta+1 / \eta^{\prime}=1$ and Theorem 6.1 we have

$$
\begin{aligned}
f_{B}\left|F_{1}(y)\right| d y & \leq\left(f_{B}\left|b(y)-b_{2 B}\right|^{\eta^{\prime}} d y\right)^{1 / \eta^{\prime}}\left(f_{B}\left|I_{\rho} f(y)\right|^{\eta} d y\right)^{1 / \eta} \\
& =\frac{1}{\psi(t)}\left(f_{B}\left|b(y)-b_{2 B}\right|^{\eta^{\prime}} d y\right)^{1 / \eta^{\prime}}\left(\psi(t)^{\eta} f_{B}\left|I_{\rho} f(y)\right|^{\eta} d y\right)^{1 / \eta} \\
& \lesssim\|b\|_{\mathcal{L}_{1, \psi}}\left(M_{\psi^{\eta}}\left(\left|I_{\rho} f\right|^{\eta}\right)(x)\right)^{1 / \eta} .
\end{aligned}
$$

Choose $v \in(1, \eta)$ such that $n / v-\epsilon / 2 \geq n-\epsilon$. Then by the almost decreasingness of $r \mapsto \rho(r) / r^{n-\epsilon}$ we have the almost decreasingness of $r \mapsto \rho(r) / r^{n / v-\epsilon / 2}$. Hence, from Corollary 3.6 it follows that there exists an $N$-function $\Psi$ such that $I_{\rho}$ is bounded from $L^{v}\left(\mathbb{R}^{n}\right)$ to $L^{\Psi}\left(\mathbb{R}^{n}\right)$. Let $\widetilde{\Psi}$ be the complementary function of $\Psi$. Then by the generalized Hölder's inequality 4.2 , 4.3), (3.3) and the boundedness of $I_{\rho}$ we have

$$
\begin{aligned}
f_{B}\left|F_{2}(y)\right| d y & \leq \frac{2}{|B|}\left\|\chi_{B}\right\|_{L^{\widetilde{\Psi}}\left(\mathbb{R}^{n}\right)}\left\|F_{2}\right\|_{L^{\Psi}\left(\mathbb{R}^{n}\right)} \\
& \lesssim \Psi^{-1}(1 /|B|)\left\|\left(b-b_{2 B}\right) f_{1}\right\|_{L^{v}\left(\mathbb{R}^{n}\right)} \\
& \lesssim \frac{\rho^{*}(t)}{|B|^{1 / v}}\left\|\left(b-b_{2 B}\right) f\right\|_{L^{v}(2 B)} .
\end{aligned}
$$

Let $1 / v=1 / u+1 / \eta$. Then by Hölder's inequality and Theorem 6.1 we have

$$
\begin{aligned}
f_{B}\left|F_{2}(y)\right| d y & \lesssim \rho^{*}(t)\left(f_{2 B}\left|b(y)-b_{2 B}\right|^{u} d y\right)^{1 / u}\left(f_{2 B}|f(y)|^{\eta} d y\right)^{1 / \eta} \\
& \lesssim \frac{1}{\psi(2 t)}\left(f_{2 B}\left|b(y)-b_{2 B}\right|^{u} d y\right)^{1 / u}\left(\left(\rho^{*}(2 t) \psi(2 t)\right)^{\eta} f_{2 B}|f(y)|^{\eta} d y\right)^{1 / \eta} \\
& \lesssim\|b\|_{\mathcal{L}_{1, \psi}}\left(M_{\left(\rho^{*} \psi\right)^{\eta}}\left(|f|^{\eta}\right)(x)\right)^{1 / \eta} .
\end{aligned}
$$

Finally, using the relation

$$
\frac{1}{2} \leq \frac{|y-z|}{|x-z|} \leq 2 \text { for } y \in B \text { and } z \notin 2 B
$$

and 3.13 , we have

$$
\begin{aligned}
\left|F_{3}(y)\right| & =\left|I_{\rho}\left(\left(b-b_{2 B}\right) f_{2}\right)(y)-I_{\rho}\left(\left(b-b_{2 B}\right) f_{2}\right)(x)\right| \\
& =\left|\int_{\mathbb{R}^{n}}\left(\frac{\rho(|y-z|)}{|y-z|^{n}}-\frac{\rho(|x-z|)}{|x-z|^{n}}\right)\left(b(z)-b_{2 B}\right) f_{2}(z) d z\right| \\
& \lesssim \int_{\mathbb{R}^{n} \backslash 2 B} \frac{|x-y| \rho^{*}(|x-z|)}{|x-z|^{n+1}}\left|b(z)-b_{2 B}\right||f(z)| d z \\
& =\sum_{j=0}^{\infty} \int_{2^{j+2} B \backslash 2^{j+1} B} \frac{|x-y| \rho^{*}(|x-z|)}{|x-z|^{n+1}}\left|b(z)-b_{2 B}\right||f(z)| d z .
\end{aligned}
$$


By the doubling condition of $\rho^{*}$ (see Remark 4.3), Hölder's inequality and Lemma 6.3 we have

$$
\begin{aligned}
& \int_{2^{j+2} B \backslash 2^{j+1} B} \frac{|x-y| \rho^{*}(|x-z|)}{|x-z|^{n+1}}\left|b(z)-b_{2 B}\right||f(z)| d z \\
\lesssim & \frac{t \rho^{*}\left(2^{j+2} t\right)}{\left(2^{j+2} t\right)^{n+1}} \int_{2^{j+2} B \backslash 2^{j+1} B}\left|b(z)-b_{2 B}\right||f(z)| d z \\
\lesssim & \frac{\rho^{*}\left(2^{j+2} t\right)}{2^{j+2}}\left(f_{2^{j+2} B}\left|b(z)-b_{2 B}\right|^{\eta^{\prime}} d z\right)^{1 / \eta^{\prime}}\left(f_{2^{j+2} B}|f(z)|^{\eta} d z\right)^{1 / \eta} \\
\leq & \frac{j+2}{2^{j+2}}\|b\|_{\mathcal{L}_{1, \psi}}\left(\left(\rho^{*}\left(2^{j+2} t\right) \psi\left(2^{j+2} t\right)\right)^{\eta} f_{2^{j+2} B}|f(z)|^{\eta} d z\right)^{1 / \eta} .
\end{aligned}
$$

Then

$$
\begin{aligned}
\left|F_{3}(y)\right| & \lesssim\|b\|_{\mathcal{L}_{1, \psi}} \sum_{j=0}^{\infty} \frac{j+2}{2^{j+2}}\left(\left(\rho^{*}\left(2^{j+2} t\right) \psi\left(2^{j+2} t\right)\right)^{\eta} f_{2^{j+2} B}|f(z)|^{\eta} d z\right)^{1 / \eta} \\
& \lesssim\|b\|_{\mathcal{L}_{1, \psi}}\left(M_{\left(\rho^{*} \psi\right)^{\eta}}\left(|f|^{\eta}\right)(x)\right)^{1 / \eta}
\end{aligned}
$$

which shows

$$
f_{B}\left|F_{3}(y)\right| d y \lesssim\|b\|_{\mathcal{L}_{1, \psi}}\left(M_{\left(\rho^{*} \psi\right)^{\eta}}\left(|f|^{\eta}\right)(x)\right)^{1 / \eta}
$$

Therefore, we have 6.1 and the conclusion.

Next we define the dyadic maximal operator $M^{\text {dy }}$. We denote by $\mathcal{Q}^{\text {dy }}$ the set of all dyadic cubes, that is,

$$
\mathcal{Q}^{\mathrm{dy}}=\left\{Q_{j, k}=\prod_{i=1}^{n}\left[2^{-j} k_{i}, 2^{-j}\left(k_{i}+1\right)\right): j \in \mathbb{Z}, k=\left(k_{1}, \ldots, k_{n}\right) \in \mathbb{Z}^{n}\right\} .
$$

Then we define

$$
M^{\mathrm{dy}} f(x)=\sup _{R \in \mathcal{Q}^{\mathrm{dy}}, R \ni x} f_{R}|f(y)| d y, \quad x \in \mathbb{R}^{n},
$$

where the supremum is taken over all $R \in \mathcal{Q}^{\text {dy }}$ containing $x$.

Next we prove the following proposition.

Proposition 6.4. Let $\Phi \in \Delta_{2}$. If $M^{\mathrm{dy}} f \in L^{\Phi}\left(\mathbb{R}^{n}\right)$, then

$$
\left\|M^{\mathrm{dy}} f\right\|_{L^{\Phi}} \leq C\left\|M^{\sharp} f\right\|_{L^{\Phi}},
$$

where $C$ is a positive constant which is dependent only on $n$ and $\Phi$.

The following lemma is well known as the good lambda inequality, see 7, Theorem 3.4.4.] for example. 
Lemma 6.5. For all $\gamma>0$, all $\lambda>0$, and all locally integrable functions $f$ on $\mathbb{R}^{n}$, the following estimate holds

$$
\left|\left\{x \in \mathbb{R}^{n}: M^{\mathrm{dy}} f(x)>2 \lambda, M^{\sharp} f(x) \leq \gamma \lambda\right\}\right| \leq 2^{n} \gamma\left|\left\{x \in \mathbb{R}^{n}: M^{\mathrm{dy}} f(x)>\lambda\right\}\right| .
$$

Proof of Proposition 6.4. For a positive real number $N$ we set

$$
I_{N}=\int_{0}^{N} \Phi^{\prime}(\lambda)\left|\left\{x \in \mathbb{R}^{n}: M^{\mathrm{dy}} f(x)>\lambda\right\}\right| d \lambda .
$$

We note that $I_{N} \leq \int_{\mathbb{R}^{n}} \Phi\left(M^{\text {dy }} f(x)\right) d x<\infty$. By Lemma 4.4 we have

$$
\begin{aligned}
I_{N} & =2 \int_{0}^{N / 2} \Phi^{\prime}(2 \lambda)\left|\left\{x \in \mathbb{R}^{n}: M^{\mathrm{dy}} f(x)>2 \lambda\right\}\right| d \lambda \\
& \leq 2 C_{\Phi} \int_{0}^{N / 2} \Phi^{\prime}(\lambda)\left|\left\{x \in \mathbb{R}^{n}: M^{\mathrm{dy}} f(x)>2 \lambda\right\}\right| d \lambda .
\end{aligned}
$$

Then, using the good lambda inequality, we obtain the following sequence of inequalities:

$$
\begin{aligned}
I_{N} \leq & 2 C_{\Phi} \int_{0}^{N / 2} \Phi^{\prime}(\lambda)\left|\left\{x \in \mathbb{R}^{n}: M^{\mathrm{dy}} f(x)>2 \lambda, M^{\sharp} f(x) \leq \gamma \lambda\right\}\right| d \lambda \\
& +2 C_{\Phi} \int_{0}^{N / 2} \Phi^{\prime}(\lambda)\left|\left\{x \in \mathbb{R}^{n}: M^{\sharp} f(x)>\gamma \lambda\right\}\right| d \lambda \\
\leq & 2^{n+1} C_{\Phi} \gamma \int_{0}^{N / 2} \Phi^{\prime}(\lambda)\left|\left\{x \in \mathbb{R}^{n}: M^{\mathrm{dy}} f(x)>\lambda\right\}\right| d \lambda \\
& +2 C_{\Phi} \int_{0}^{N / 2} \Phi^{\prime}(\lambda)\left|\left\{x \in \mathbb{R}^{n}: M^{\sharp} f(x)>\gamma \lambda\right\}\right| d \lambda \\
\leq & 2^{n+1} C_{\Phi} \gamma I_{N}+2 C_{\Phi} \frac{1}{\gamma} \int_{0}^{N \gamma / 2} \Phi^{\prime}(\lambda / \gamma)\left|\left\{x \in \mathbb{R}^{n}: M^{\sharp} f(x)>\lambda\right\}\right| d \lambda .
\end{aligned}
$$

At this point we let $2^{n+1} C_{\Phi} \gamma=1 / 2$. Since $I_{N}$ is finite, we can subtract from both sides of the inequality the quantity $I_{N} / 2$ to obtain

$$
\begin{aligned}
I_{N} & \leq 2^{n+4} C_{\Phi}^{2} \int_{0}^{N /\left(2^{n+3} C_{\Phi}\right)} \Phi^{\prime}\left(2^{n+2} C_{\Phi} \lambda\right)\left|\left\{x \in \mathbb{R}^{n}: M^{\sharp} f(x)>\lambda\right\}\right| d \lambda \\
& \leq C_{n, \Phi} \int_{0}^{\infty} \Phi^{\prime}(\lambda)\left|\left\{x \in \mathbb{R}^{n}: M^{\sharp} f(x)>\lambda\right\}\right| d \lambda,
\end{aligned}
$$

where $C_{n, \Phi}$ is a constant dependent only on $n$ and $\Phi$, from which we obtain

$$
\int_{\mathbb{R}^{n}} \Phi\left(M^{\mathrm{dy}} f(x)\right) d x \leq C_{n, \Phi} \int_{\mathbb{R}^{n}} \Phi\left(M^{\sharp} f(x)\right) d x .
$$

This shows 6.2. 


\section{Proof of Theorem 3.13}

We first note that, for $\theta \in(0, \infty)$,

$$
\left\||g|^{\theta}\right\|_{L^{\Phi}}=\left(\|g\|_{L^{\left.\Phi((\cdot))^{\theta}\right)}}\right)^{\theta} .
$$

Lemma 7.1. Under the assumption in Theorem 3.13(i), if $f \in L_{\mathrm{comp}}^{\infty}\left(\mathbb{R}^{n}\right)$, then $I_{\rho} f \in$ $L^{\Psi}\left(\mathbb{R}^{n}\right)$.

Proof. If $f \in L_{\text {comp }}^{\infty}\left(\mathbb{R}^{n}\right)$, then $f \in L^{\Phi}\left(\mathbb{R}^{n}\right)$, since $L_{\text {comp }}^{\infty}\left(\mathbb{R}^{n}\right) \subset L^{\Phi}\left(\mathbb{R}^{n}\right)$. By (3.11) and Theorem 3.1. $I_{\rho}$ is bounded from $L^{\Phi}\left(\mathbb{R}^{n}\right)$ to $L^{\Theta}\left(\mathbb{R}^{n}\right)$. Then $I_{\rho} f$ is in $L^{\Theta}\left(\mathbb{R}^{n}\right)$. On the other hand, since $r \mapsto \rho(r) / r^{n}$ is almost decreasing, if the support of $f$ is in $B(0, R)$, then

$$
\left|I_{\rho} f(x)\right| \leq\|f\|_{L^{\infty}} \int_{B(0, R)} \frac{\rho(|x-y|)}{|x-y|^{n}} d y \lesssim\|f\|_{L^{\infty}} \int_{0}^{R} \frac{\rho(t)}{t} d t<\infty .
$$

Then $I_{\rho} f$ is in $L^{\Theta}\left(\mathbb{R}^{n}\right) \cap L^{\infty}\left(\mathbb{R}^{n}\right)$.

Next, by 3.12 and the almost increasingness of $\psi$ we have

$$
\Theta^{-1}\left(1 / r^{n}\right) \lesssim \frac{\Psi^{-1}\left(1 / r^{n}\right)}{\psi(r)} \lesssim \frac{\Psi^{-1}\left(1 / r^{n}\right)}{\psi(1)} \quad \text { for } r \geq 1
$$

and then

$$
\Theta^{-1}(u) \lesssim \Psi^{-1}(u) \quad \text { for } u \leq 1
$$

Hence, we conclude that

$$
\Psi(t) \leq \begin{cases}\Theta(C t) & \text { if } t \leq 1 \\ \infty & \text { if } t>1\end{cases}
$$

which shows that $L^{\Theta}\left(\mathbb{R}^{n}\right) \cap L^{\infty}\left(\mathbb{R}^{n}\right) \subset L^{\Psi}\left(\mathbb{R}^{n}\right)$.

Proof of Theorem 3.13(i). We may assume that $\Phi, \Psi \in \Delta_{2} \cap \nabla_{2}$ and $\Theta \in \nabla_{2}$. We may also assume that $b$ is real valued, since the commutator $\left[b, I_{\rho}\right] f$ is linear with respect to $b$ and $\|\Re(b)\|_{\mathcal{L}_{1, \psi}},\|\Im(b)\|_{\mathcal{L}_{1, \psi}} \leq\|b\|_{\mathcal{L}_{1, \psi}}$. Let

$$
b_{k}(x)= \begin{cases}k & \text { if } b(x)>k, \\ b(x) & \text { if }-k \leq b(x) \leq k, \\ -k & \text { if } b(x)<-k .\end{cases}
$$

Then $b_{k} \in L^{\infty}\left(\mathbb{R}^{n}\right)$ and $\left\|b_{k}\right\|_{\mathcal{L}_{1, \psi}} \leq(9 / 4)\|b\|_{\mathcal{L}_{1, \psi}}$. For $f \in C_{\text {comp }}^{\infty}\left(\mathbb{R}^{n}\right), b_{k} f$ lies in $L_{\text {comp }}^{\infty}\left(\mathbb{R}^{n}\right)$, thus $I_{\rho}\left(b_{k} f\right)$ lies in $L^{\Psi}\left(\mathbb{R}^{n}\right)$ by Lemma 7.1 . Likewise, $b_{k} I_{\rho} f$ also lies in $L^{\Psi}\left(\mathbb{R}^{n}\right)$. Since 
$\Psi \in \nabla_{2}, M^{\mathrm{dy}}\left[b, I_{\rho}\right] f$ is also in $L^{\Psi}\left(\mathbb{R}^{n}\right)$. From this fact and Propositions 6.2 and 6.4 it follows that

$$
\begin{aligned}
\left\|\left[b_{k}, I_{\rho}\right] f\right\|_{L^{\Psi}} & \leq\left\|M^{\mathrm{dy}}\left(\left[b_{k}, I_{\rho}\right] f\right)\right\|_{L^{\Psi}} \lesssim\left\|M^{\sharp}\left(\left[b_{k}, I_{\rho}\right] f\right)\right\|_{L^{\Psi}} \\
& \lesssim\|b\|_{\mathcal{L}_{1, \psi}}\left(\left\|\left(M_{\psi^{\eta}}\left(\left|I_{\rho} f\right|^{\eta}\right)\right)^{1 / \eta}\right\|_{L^{\Psi}}+\left\|\left(M_{\left(\rho^{*} \psi\right)^{\eta}}\left(|f|^{\eta}\right)\right)^{1 / \eta}\right\|_{L^{\Psi}}\right)
\end{aligned}
$$

here, we can choose $\eta \in(1, \infty)$ such that $\Phi\left((\cdot)^{1 / \eta}\right), \Psi\left((\cdot)^{1 / \eta}\right)$ and $\Theta\left((\cdot)^{1 / \eta}\right)$ are in $\bar{\nabla}_{2}$ by Lemma 4.5. We show that

$$
\left\|\left(M_{\psi^{\eta}}\left(\left|I_{\rho} f\right|^{\eta}\right)\right)^{1 / \eta}\right\|_{L^{\Psi}}+\left\|\left(M_{\left(\rho^{*} \psi\right)^{\eta}}\left(|f|^{\eta}\right)\right)^{1 / \eta}\right\|_{L^{\Psi}} \lesssim\|f\|_{L^{\Phi}},
$$

where we note that $\psi^{\eta}$ and $\left(\rho^{*} \psi\right)^{\eta}$ are almost increasing.

By Theorems 3.1 and 3.8 we see that $I_{\rho}$ is bounded from $L^{\Phi}\left(\mathbb{R}^{n}\right)$ to $L^{\Theta}\left(\mathbb{R}^{n}\right)$ and $M_{\psi^{\eta}}$ is bounded from $L^{\Theta\left((\cdot)^{1 / \eta}\right)}\left(\mathbb{R}^{n}\right)$ to $L^{\Psi\left((\cdot)^{1 / \eta}\right)}\left(\mathbb{R}^{n}\right)$, respectively. Then, using $(7.1)$, we have

$$
\begin{aligned}
\left\|\left(M_{\psi^{\eta}}\left(\left|I_{\rho} f\right|^{\eta}\right)\right)^{1 / \eta}\right\|_{L^{\Psi}} & =\left(\left\|M_{\psi^{\eta}}\left(\left|I_{\rho} f\right|^{\eta}\right)\right\|_{L^{\Psi\left((\cdot)^{1 / \eta}\right)}}\right)^{1 / \eta} \\
& \lesssim\left(\left\|\left|I_{\rho} f\right|^{\eta}\right\|_{L^{\left.\Theta((\cdot))^{1 / \eta}\right)}}\right)^{1 / \eta}=\left\|I_{\rho} f\right\|_{L^{\Theta}} \lesssim\|f\|_{L^{\Phi}} .
\end{aligned}
$$

From 3.11 and 3.12 it follows that

$$
\left(\rho^{*}(r) \psi(r)\right)^{\eta}\left(\Phi^{-1}\left(1 / r^{n}\right)\right)^{\eta} \leq A^{2 \eta}\left(\Psi^{-1}\left(1 / r^{n}\right)\right)^{\eta} .
$$

By using Theorem 3.8, we have the boundedness of $M_{\left(\rho^{*} \psi\right)^{\eta}}$ from $L^{\Phi\left((\cdot)^{1 / \eta}\right)}$ to $L^{\Psi\left((\cdot)^{1 / \eta}\right)}$. That is,

$$
\left\|\left(M_{\left(\rho^{*} \psi\right)^{\eta}}\left(|f|^{\eta}\right)\right)^{1 / \eta}\right\|_{L^{\Psi}}=\left(\left\|M_{\left(\rho^{*} \psi\right)^{\eta}}\left(|f|^{\eta}\right)\right\|_{L^{\Psi\left((\cdot)^{1 / \eta}\right)}}\right)^{1 / \eta} \lesssim\left(\left\||f|^{\eta}\right\|_{L^{\Phi\left((\cdot)^{1 / \eta}\right)}}\right)^{1 / \eta}=\|f\|_{L^{\Phi}}
$$

Therefore, we obtain

$$
\left\|\left[b_{k}, I_{\rho}\right] f\right\|_{L^{\Psi}} \lesssim\|b\|_{\mathcal{L}_{1, \psi}}\|f\|_{L^{\Phi}} \quad \text { for all } f \in C_{\mathrm{comp}}^{\infty}\left(\mathbb{R}^{n}\right) .
$$

By the standard argument (see [7, p. 240] for example) we deduce that, for some subsequence of integers $k_{j},\left[b_{k_{j}}, I_{\rho}\right] f \rightarrow\left[b, I_{\rho}\right] f$ a.e. Letting $j \rightarrow \infty$ and using Fatou's lemma, we have

$$
\left\|\left[b, I_{\rho}\right] f\right\|_{L^{\Psi}} \lesssim\|b\|_{\mathcal{L}_{1, \psi}}\|f\|_{L^{\Phi}} \quad \text { for all } f \in C_{\text {comp }}^{\infty}\left(\mathbb{R}^{n}\right) .
$$

Since $C_{\text {comp }}^{\infty}\left(\mathbb{R}^{n}\right)$ is dense in $L^{\Phi}\left(\mathbb{R}^{n}\right)$ (see Remark 2.8(ii)), it follows that the commutator admits a bounded extension on $L^{\Phi}\left(\mathbb{R}^{n}\right)$ that satisfies 3.14 .

Proof of Theorem 3.13(ii). We use the method by Janson [10]. Since $|z|^{n-\alpha}$ is infinitely differentiable in an open set, we may choose $z_{0} \neq 0$ and $\delta>0$ such that $|z|^{n-\alpha}$ can be expressed in the neighborhood $\left|z-z_{0}\right|<2 \delta$ as an absolutely convergent Fourier series, $|z|^{n-\alpha}=\sum a_{j} e^{i v_{j} \cdot z}$. (The exact form of the vectors $v_{j}$ is irrelevant.) 
Set $z_{1}=z_{0} / \delta$. If $\left|z-z_{1}\right|<2$, we have the expansion

$$
|z|^{n-\alpha}=\delta^{-n+\alpha}|\delta z|^{n-\alpha}=\delta^{-n+\alpha} \sum a_{j} e^{i v_{j} \cdot \delta z} .
$$

Choose now any ball $B=B\left(x_{0}, r\right)$. Set $y_{0}=x_{0}-r z_{1}$ and $B^{\prime}=B\left(y_{0}, r\right)$. Then, if $x \in B$ and $y \in B^{\prime}$,

$$
\left|\frac{x-y}{r}-z_{1}\right| \leq\left|\frac{x-x_{0}}{r}\right|+\left|\frac{y-y_{0}}{r}\right|<2 .
$$

Denote $\operatorname{sgn}\left(f(x)-f_{B^{\prime}}\right)$ by $s(x)$. Then

$$
\begin{aligned}
\int_{B}\left|b(x)-b_{B^{\prime}}\right| d x & =\int_{B}\left(b(x)-b_{B^{\prime}}\right) s(x) d x=\frac{1}{\left|B^{\prime}\right|} \int_{B} \int_{B^{\prime}}(b(x)-b(y)) s(x) d y d x \\
& =\frac{1}{\left|B^{\prime}\right|} \int_{\mathbb{R}^{n}} \int_{\mathbb{R}^{n}}(b(x)-b(y)) \frac{r^{n-\alpha}\left|\frac{x-y}{r}\right|^{n-\alpha}}{|x-y|^{n-\alpha}} s(x) \chi_{B}(x) \chi_{B^{\prime}}(y) d y d x \\
& =\frac{r^{n-\alpha} \delta^{-n+\alpha}}{\left|B^{\prime}\right|} \int_{\mathbb{R}^{n}} \int_{\mathbb{R}^{n}} \frac{b(x)-b(y)}{|x-y|^{n-\alpha}} \sum a_{j} e^{i v_{j} \cdot \delta \frac{x-y}{r}} s(x) \chi_{B}(x) \chi_{B^{\prime}}(y) d y d x .
\end{aligned}
$$

Here, we set $C=\delta^{-n+\alpha}|B(0,1)|^{-1}$ and

$$
g_{j}(y)=e^{-i v_{j} \cdot \delta \frac{y}{r}} \chi_{B^{\prime}}(y), \quad h_{j}(x)=e^{i v_{j} \cdot \delta \frac{x}{r}} s(x) \chi_{B}(x) .
$$

Then

$$
\begin{aligned}
\int_{B}\left|b(x)-b_{B^{\prime}}\right| d x & =C r^{-\alpha} \sum a_{j} \int_{\mathbb{R}^{n}} \int_{\mathbb{R}^{n}} \frac{b(x)-b(y)}{|x-y|^{n-\alpha}} g_{j}(y) h_{j}(x) d y d x \\
& =C r^{-\alpha} \sum a_{j} \int_{\mathbb{R}^{n}}\left(\left[b, I_{\alpha}\right] g_{j}\right)(x) h_{j}(x) d x \\
& \leq C r^{-\alpha} \sum\left|a_{j}\right| \int_{\mathbb{R}^{n}}\left|\left(\left[b, I_{\alpha}\right] g_{j}\right)(x)\right|\left|h_{j}(x)\right| d x \\
& =C r^{-\alpha} \sum\left|a_{j}\right| \int_{B}\left|\left(\left[b, I_{\alpha}\right] g_{j}\right)(x)\right| d x \\
& \leq 2 C r^{-\alpha} \sum\left|a_{j}\right|\left\|\chi_{B}\right\|_{L^{\widetilde{\Psi}}}\left\|\left[b, I_{\alpha}\right] g_{j}\right\|_{L^{\Psi}} \\
& \leq 2 C r^{-\alpha}\left\|\left[b, I_{\alpha}\right]\right\|_{L^{\Phi} \rightarrow L^{\Psi}}|B| \Psi^{-1}\left(|B|^{-1}\right) \sum\left|a_{j}\right|\left\|g_{j}\right\|_{L^{\Phi}} .
\end{aligned}
$$

Since $\left\|g_{j}\right\|_{L^{\Phi}}=\left\|\chi_{B^{\prime}}\right\|_{L^{\Phi}}=1 / \Phi^{-1}\left(\left|B^{\prime}\right|^{-1}\right) \sim 1 / \Phi^{-1}\left(r^{-n}\right)$, we have

$$
\frac{1}{\psi(B)} f_{B}\left|b(x)-b_{B^{\prime}}\right| d x \lesssim\left\|\left[b, I_{\alpha}\right]\right\|_{L^{\Phi} \rightarrow L^{\Psi}} \frac{\Psi^{-1}\left(r^{-n}\right)}{r^{\alpha} \psi(B) \Phi^{-1}\left(r^{-n}\right)} \lesssim\left\|\left[b, I_{\alpha}\right]\right\|_{L^{\Phi} \rightarrow L^{\Psi}} .
$$

That is, $\|b\|_{\mathcal{L}_{1, \psi}} \lesssim\left\|\left[b, I_{\alpha}\right]\right\|_{L^{\Phi} \rightarrow L^{\Psi}}$ and we have the conclusion.

\section{Acknowledgments}

The authors would like to thank the referee for her/his careful reading and useful comments. This research was supported by Grant-in-Aid for Scientific Research (B), No. 15H03621, Japan Society for the Promotion of Science. 


\section{References}

[1] R. Arai and E. Nakai, Commutators of Calderón-Zygmund and generalized fractional integral operators on generalized Morrey spaces, Rev. Mat. Complut. 31 (2018), no. 2, $287-331$.

[2] S. Chanillo, A note on commutators, Indiana Univ. Math. J. 31 (1982), no. 1, 7-16.

[3] A. Cianchi, Strong and weak type inequalities for some classical operators in Orlicz spaces, J. London Math. Soc. (2) 60 (1999), no. 1, 187-202.

[4] F. Deringoz, V. S. Guliyev, E. Nakai, Y. Sawano and M. Shi, Generalized fractional maximal and integral operators on Orlicz and generalized Orlicz-Morrey spaces of the third kind, Positivity, Online First.

https://link.springer.com/article/10.1007/s11117-018-0635-9

https://arxiv.org/abs/1812.03649

[5] D. E. Edmunds, P. Gurka and B. Opic, Double exponential integrability of convolution operators in generalized Lorentz-Zygmund spaces, Indiana Univ. Math. J. 44 (1995), no. $1,19-43$.

[6] X. Fu, D. Yang and W. Yuan, Generalized fractional integrals and their commutators over non-homogeneous metric measure spaces, Taiwanese J. Math. 18 (2014), no. 2, 509-557.

[7] L. Grafakos, Modern Fourier Analysis, Third edition, Graduate Texts in Mathematics 250, Springer, New York, 2014.

[8] V. S. Guliyev, F. Deringoz and S. G. Hasanov, Riesz potential and its commutators on Orlicz spaces, J. Inequal. Appl. 2017 (2017), no. 75, 18 pp.

[9] L. I. Hedberg, On certain convolution inequalities, Proc. Amer. Math. Soc. 36 (1972), $505-510$.

[10] S. Janson, Mean oscillation and commutators of singular integral operators, Ark. Mat. 16 (1978), no. 2, 263-270.

[11] R. Kawasumi and E. Nakai, Pointwise multipliers on weak Orlicz spaces, preprint. https://arxiv.org/abs/1811.02858

[12] H. Kita, On maximal functions in Orlicz spaces, Proc. Amer. Math. Soc. 124 (1996), no. 10, 3019-3025. 
[13] _ On Hardy-Littlewood maximal functions in Orlicz spaces, Math. Nachr. 183 (1997), 135-155.

[14] Orlicz spaces and their applications (Japanese), Iwanami Shoten, Publishers, Tokyo, 2009.

[15] V. Kokilashvili and M. Krbec, Weighted Inequalities in Lorentz and Orlicz Spaces, World Scientific, River Edge, NJ, 1991.

[16] M, A. Krasnoselsky and Y. B. Rutitsky, Convex functions and Orlicz spaces, Translated from the first Russian edition by Leo F. Boron. P. Noordhoff Ltd., Groningen, 1961.

[17] L. Maligranda, Orlicz Spaces and Interpolation, Seminars in Mathematics 5, Universidade Estadual de Campinas, Departamento de Matemática, Campinas, 1989.

[18] Y. Mizuta, E. Nakai, T. Ohno and T. Shimomura, Boundedness of fractional integral operators on Morrey spaces and Sobolev embeddings for generalized Riesz potentials, J. Math. Soc. Japan 62 (2010), no. 3, 707-744.

[19] E. Nakai, On generalized fractional integrals in the Orlicz spaces, in: Proceedings of the Second ISAAC Congress, Vol. 1 (Fukuoka, 1999), 75-81, Int. Soc. Anal. Appl. Comput. 7, Kluwer Acad. Publ., Dordrecht, 2000.

[20] _ _ On generalized fractional integrals, Taiwanese J. Math. 5 (2001), no. 3, 587602.

[21] _ On generalized fractional integrals in the Orlicz spaces on spaces of homogeneous type, Sci. Math. Jpn. 54 (2001), no. 3, 473-487.

[22] _ On generalized fractional integrals on the weak Orlicz spaces, $\mathrm{BMO}_{\phi}$, the Morrey spaces and the Campanato spaces, in: Function Spaces, Interpolation Theory and Related Topics (Lund, 2000), 389-401, de Gruyter, Berlin, 2002.

[23] _ Generalized fractional integrals on Orlicz-Morrey spaces, in: Banach and Function Spaces, 323-333, Yokohama Publishers, Yokohama, 2004.

[24] - Orlicz-Morrey spaces and the Hardy-Littlewood maximal function, Studia Math. 188 (2008), no. 3, 193-221.

[25] _ A generalization of Hardy spaces $H^{p}$ by using atoms, Acta Math. Sin. (Engl. Ser.) 24 (2008), no. 8, 1243-1268. 
[26] E. Nakai and H. Sumitomo, On generalized Riesz potentials and spaces of some smooth functions, Sci. Math. Jpn. 54 (2001), no. 3, 463-472.

[27] R. O'Neil, Fractional integration in Orlicz spaces I, Trans. Amer. Math. Soc. 115 (1965), 300-328.

[28] W. Orlicz, Über eine gewisse Klasse von Räumen vom Typus B, Bull. Acad. Polonaise A (1932), 207-220; reprinted in his Collected Papers, PWN, Warszawa 1988, 217-230.

[29]_ _ Über Räume $\left(L^{M}\right)$, Bull. Acad. Polonaise A (1936), 93-107; reprinted in his Collected Papers, PWN, Warszawa 1988, 345-359.

[30] M. M. Rao and Z. D. Ren, Theory of Orlicz Spaces, Monographs and Textbooks in Pure and Applied Mathematics 146, Marcel Dekker, New York, 1991.

[31] Y. Sawano, S. Sugano and H. Tanaka, Generalized fractional integral operators and fractional maximal operators in the framework of Morrey spaces, Trans. Amer. Math. Soc. 363 (2011), no. 12, 6481-6503.

[32] R. S. Strichartz, A note on Trudinger's extension of Sobolev's inequalities, Indiana Univ. Math. J. 21 (1972), 841-842.

[33] A. Torchinsky, Interpolation of operations and Orlicz classes, Studia Math. 59 (1976), no. 2, 177-207.

[34] N. S. Trudinger, On imbeddings into Orlicz spaces and some applications, J. Math. Mech. 17 (1967), 473-483.

[35] G. Weiss, A note on Orlicz spaces, Portugal. Math. 15 (1956), 35-47.

Minglei Shi

Department of Mathematics, Ibaraki University, Mito, Ibaraki 310-8512, Japan

E-mail address: 18nd2061@vc.ibaraki.ac.jp, stfoursml@gmail.com

Ryutaro Arai

Department of Mathematics, Ibaraki University, Mito, Ibaraki 310-8512, Japan

E-mail address: 18nd201t@vc.ibaraki.ac.jp, araryu314159@gmail.com

Eiichi Nakai

Department of Mathematics, Ibaraki University, Mito, Ibaraki 310-8512, Japan

E-mail address: eiichi.nakai.math@vc.ibaraki.ac.jp 